\title{
Extraction of Pectin from Orange Peels and Characterizing Its Physical and Chemical Properties
}

\author{
Enkuahone Abebe Alamineh \\ Department of Chemical Engineering, Debre Berhan University, Debre Berhan, Ethiopia \\ Email address: \\ enkuahone@dbu.edu.et, enkush2014@gmail.com, enkuahone1883@gmail.com

\section{To cite this article:} \\ Enkuahone Abebe Alamineh. Extraction of Pectin from Orange Peels and Characterizing Its Physical and Chemical Properties. American \\ Journal of Applied Chemistry. Vol. 6, No. 2, 2018, pp. 51-56. doi: 10.11648/j.ajac.20180602.13
}

Received: March 30, 2018; Accepted: April 19, 2018; Published: April 26, 2018

\begin{abstract}
The aim of this study was to extract pectin from orange peels. In order to increase profits for citrus orange growers and processors, citrus orange peels, a by-product of citrus orange processing, were investigated as a source of pectin. An orange, specifically, the sweet orange is the most commonly grown tree orange in Ethiopia. The present work addressed to the development of the part of the process needed for the extraction of value added products like pectin from orange peel, which is the waste of orange juice processing industry. The outcome of the present work highlighted that the sweet orange peels are good source of pectin and does have the potential to become important raw material for food processing industries. It was found from the experimental observations that the peel source. It was concluded that the process in which pectin was first extracted using technique of water bathing or drying followed by acid extraction of pectin was most suitable for industrial production for isolation of pectin. These results demonstrated the pectin, providing potential benefits for industrial extraction of pectin from an economic and environmental point of view. It was possible that pectin loss was occurring during the precipitation step and the alcohol washes. There was a large cloudy mass within the liquid that was possibly pectin that was not being recovered and retained in the following separation and washing steps. Experiments were set up to test the method of retrieving the pectin from the alcohol after precipitation. Experiments were conducted under standard extraction conditions comparing centrifugation and cheesecloth as retrieval methods for the precipitated pectin. The pectin yields for the centrifugation and cheesecloth methods were $14.3 \%$ and $10.6 \%$, respectively. These results indicated that more pectin was retained using the centrifugation method.
\end{abstract}

Keywords: Pectin, Citrus Orange, Peel, Centrifugation

\section{Introduction}

Pectin can be used to improve the mouth feel and the pulp stability in juice based drinks and as a stabilizer in acidic protein beverages. Pectin also reduces syneresis in jams and marmalades and increases the gel strength of low calorie jams. Pectin is used in confectionery jellies to give a good gel structure and a clean bite. The typical dosage of pectin in food applications is between 0.5 up to $1 \%$. Besides its use in the food industry, pectin is also used in pharmaceutical and cosmetic products. [1]

Pectin is a natural product which can be found in the cell walls of all higher plants and it has long been used for its gel formation, thickening and stabilizing properties in a wide range of applications from food to the pharmaceutical and cosmetic industries. When an orange juice producer is done squishing its oranges, a lot of orange peel is left unused. This peel is considered by some farmers to be waste and is may be used to feed the cattle or perhaps as fertilizer. This orange peel is the raw material for our process. By using a range of devices, pectin can be extracted from the orange peel, which can then be sold. This way the orange peel is used for a better purpose. An orange, specifically, the sweet orange (Citrus sinensis) is the most commonly grown tree orange in the Ethiopia, Orange trees are widely cultivated in tropical and subtropical climates for the sweet orange, which is peeled or cut (to avoid the bitter rind) and eaten whole, or processed to extract orange juice, $\&$ also for the fragrant peel [2]. Citrus oranges are at the top not only in total production, but also in economic value. The Albedo is the main source of pectin. Pectin includes all the esterified polygalacturonic acids at different degree of neutralization. In the presence of 
saccharine and small quantities of organic acids (usually citric acid), pectin's gelatinized, and this property is exploited by the agro chemistry and pharmaceutical industries for pectin isolation. Pectin is traditionally used as a gelling agent in a wide range of orange-based products, such as jams, marmalades, jellies, orange preparations for yoghurts and desserts and orange filling for bakery products [3]. Pectin is defined as complex mixtures of polysaccharides that make up approximately one third of the cell-wall dry substance of most types of plants. The function of pectin in plants is to contribute structural integrity to the cell wall and adhesion between cells [4]. A percentage of the galacturonic acid residues are generally esterified with methanol. The pectin's of a plant can be water-soluble, chelator soluble, or protopectins. The methods of extraction will vary based on the actual makeup for each particular plant type. For example, protopectins are brought into solution by hot dilute acids. The general makeup of the pectin content varies with ripening of the plant and it is fairly easily brought into solution depending on the plant type. After extraction pectin consists of smooth galacturonic acid regions with a few neutral sugars still attached. Commercial pectin extraction is mainly from citrus peel and apple pomace, but several other sources exist such as sugar beets and sunflower heads [5]. Extracted pectin can be categorized into two major categories depending on the percentage of galacturonic acid residues that are esterified with methanol. A degree of methoxylation (DM) greater than $50 \%$ is considered high methoxyl pectin and a DM below $50 \%$ is considered low methoxyl pectin. A subset of low methoxyl pectin exists called amidated pectin that is produced through de-desertification of high methoxyl pectin with ammonia [6].

These types of pectin can be used for a wide range of end users as their structures will yield a variety of gelling and texturizing abilities under differing conditions. Pectin is capable of forming gels with sugar and acid. Because of this gelling ability one of the well-known uses of pectin is in high sugar jams and confectionery jellies, dating back to at least the 18th century [7]. Because it is a natural additive for foods, pectin is being considered for a number of applications beyond the traditional jams and jellies. Pectin is now used as thickeners, water binders, and stabilizers. It is used in yogurts and pastry glazes and as a stabilizer in drinkable yogurts and blends of milk and orange juices [8].

Pectin is also being used as a texturizing fat replacer to mimic the mouth-feel of lipids in low-calorie foods and shorter chain galacturonic acids have been considered as clarification agents in orange juices. Pectin has also been investigated for its usefulness in the pharmaceutical industry. Among other uses it has been considered in the class of dietary fibers known to have a positive effect on digestive processes and to help lower cholesterol. It also is utilized to stabilize liquid pharmaceutical emulsions and suspensions [9].

A number of different factors are often analyzed to determine the quality and purity of pectin. The accepted measure of pectin purity is by the determination of the anhydrogalacturonic acid (AGA) content. A percentage above $65 \%$ is considered to be the typical minimum level for pectin used for various applications according to information available from the International Pectin Producers Association [4]. The purest citrus pectin contains $85-90 \%$ AGA. Another factor considered for pectin analysis is the degree of methoxylation. This measure will determine the usage of the pectin and whether it should be classified as high methoxyl (above 50\%) or low methoxyl (below 50\%). High methoxyl citrus pectin is generally at 70-80\% DM [10].

It is also of interest to determine the ash content, molecular weight, and degree of amidation for a pectin sample. Molecular weight is often determined as an indication of the gelling quality of the pectin. Amidation is sometimes desired in low methoxyl pectin because it can increase gel formation [8].

Use of Pectin Substances: The most important use of pectin substances is in the commercial and home production, of jams and jellies. Pectin's have many other uses in small amounts, which depend on their remarkable abilities to form and stabilize emulsions, in addition to the fact that they are a normal constituent of foods and may therefore be safely ingested. Pectin's have been used as thickeners and stabilizers in milk products, as chocolate milk, and as emulsifiers in cosmetics, cold creams, and soap. Pectin has also been suggested in some cases as a carrier for drugs that are injected intramuscularly. The function of pectin is to delay absorption of the drug to enable a more prolonged effect, such as in insulin-pectin preparations and pectinpenicillin preparations. Pectin and its derivatives appear also to have certain inherent therapeutic properties, particularly in certain cases of malfunction of the digestive tract. Pectin preparations have been employed as a supportive measure in cases of infant diarrhea and other alimentary tract disorders [7].

Orange composition: An orange, specifically, the sweet orange is the most commonly grown tree orange in Ethiopia. The sweet orange peels are good source of orange oil and pectin. Two methods namely simple distillation \& leaching have been explored for separation of oil from peels. When taken after extracting orange oil through simple distillation gives higher yield than leaching residue. It is concluded that the processing which orange oil is first extracted using technique of simple distillation followed by acid extraction of pectin is most suitable [10].

Pectin is widely distributed in the cell walls of the roots, stems, leaves and oranges of higher plants. It is a polysaccharide polymer compound and is the main component of dietary fibers with good gelling and emulsion stabilities. Pectin is widely used in food, pharmaceutical and chemical industries and in other specific applications. As orange waste, orange peel has high levels of pectin and can be used as raw material for pectin extraction [11].

Acid extraction method: The conventional acid extraction involves extracting the pectin using acidified water ( $\mathrm{pH}$ up to 2) and heat of the solution mixture by using water bath shaker at temperatures of $80^{\circ} \mathrm{C}$. The assembly is run for $1-2$ 
hr. duration and Pectin substances are precipitated using ethanol or isopropyl alcohol. The most commonly used acidifying materials are mineral acids including, hydrochloric, sulfuric and phosphoric acids. The acid extraction method is new technology in addition to simple to construct and operate of minimum cost [13].

Direct boiling (hot water) extraction method: A long list of various agents has been reported for the extraction of pectin from plant tissues. Extraction with the hot water is the simplest and oldest method for removing the pectin substances and have very limited amount of produced. Many organic acids and their salts such as oxalic acid, ammonium oxalate, tartaric acid, polyphosphates, and many others have been also used [10]. A very low yield of pectin obtained from dried orange peel was reported using distillation apparatus as extracting at $85-90^{\circ} \mathrm{C}$. Double extraction at $85-88^{\circ} \mathrm{C}$ for one hour using a cationic resin for the extraction of pectin from apple pomade has been reported to give higher yields and better gel strength of the product [12]. Commercially, pectin is extracted by treating the raw material with hot dilute mineral acid at $\mathrm{pH}$ (1-3). The precise length of extraction time varies with raw material, the type of pectin desired, and from one manufacturer to another. The hot pectin extract is separated from the solid residue as efficiently as possible. This is not easy since the solids are by now soft and the liquid phase are viscous. The viscosity increases with pectin concentration and molecular weight. There is a compromise between efficient extraction and solids separation and operating cost. The pectin extract may be further clarified by filtration through a filter aid. The clarified extract is then concentrated under vacuum. Powdered pectin can be produced by mixing the concentrated liquid from either apple or citrus with an alcohol (usually isopropanol). The pectin is separated as a stringy gelatinous mass, which is pressed and washed to remove the mother liquor, dried and grounded [15].

Thermo Mechanical extraction method: Has been used in locations where drying facilities and/or solvent extraction units cannot be installed. However these processes have poor yields and frequently require the use of chemical aids. Thermo mechanical extraction method also use high pressure boiler so it needs high steam consumption [12]

Microwave extraction method: During microwave heating considerable pressure builds up inside a material. The high pressure then modifies the physical properties of material tissues, breaking down the cell structure and improving the capillary porous structure of tissues. This feature allows better penetration of extracting solvent into the tissues; improving the subsequent extraction of pectin. Microwave extraction also gave a higher rate and amount of extraction [14].

Pectin manufacture is an expensive and complicated process, consisting of numerous batch-type operations, use of corrosive chemicals and expensive solvents, and filtration of exceedingly viscous solutions. The principal steps include preparation of the peel for extraction or storage for later use; removal of bitter glycosides and crude sugars; conversion of proto pectin in the peel to soluble pectin; filtration of extracted pectin; and precipitation, and drying of the prepared pectin. Citrus peel employed for pectin production is usually that from which peel oil has previously been removed. Peel is first comminuted or minced to facilitate washing and extraction. If the peel is not extracted immediately, it is heated to $104^{\circ}-108^{\circ} \mathrm{F}$. for about 10 minutes to inactivate pectin enzymes, and then dried for storage. Otherwise, inactivation of pectin enzymes is accomplished during the extraction step. The minced or dry, stored peel is washed copiously with water until all glycosides and sugars are removed. Some water-soluble pectin already in the peel may be extracted during washing, but it is of little consequence as this pectin is usually of a low jelly grade. Pectin extraction is performed by placing the prepared peel in vats of water, where the slurry is brought to a boil, and concentrated hydrochloric acid, previously diluted to prevent local overheating, is added to adjust to about $\mathrm{pH} 2.0$. Because of the strong binding power of pectin for polyvalent metal ions, extreme care must be exercised in later purifications when hydrochloric acid has been employed in order to remove the traces of lead, which are always found in this technical reagent. The acid slurry is heated at $80^{\circ}$ c for 90 minutes. During this time proto pectin is converted by "restricted hydrolysis" to pectin, which is extracted into the solution. Too high acidity or too long extraction at these temperatures may result in an undesirable degradation of the pectin. In general, several extractions of a given lot of peel under mild conditions are preferred to a single extraction under severe conditions of time, temperature, and acidity. The extract obtained may contain approximately 1 percent of pectin. If the concentration of pectin in the extract is low, it may be evaporated under low-temperature conditions to pectin content of 3 to 4 percent to facilitate subsequent precipitation. Filtration of the extract is a tedious process, because the extract is corrosive and the pectin solutions are viscous. Filtration is carried out in two stages. An initial coarse filtration removes the large pieces of pulp and other coarse material, after which a fine filtration produces a clear filtrate. Continuous drum and plate-and-frame filters, in conjunction with vacuum and pressure, are ordinarily used in the filtration procedures. The precipitated pectin is free from the solvent by pressing and/or by the action of drain or roll presses used to convey the pectin precipitate to a series of washing vats. After several washings in 50 to 70 per-cent solvent-water mixtures, the pectin is given a final dehydrating wash in 80 to 90 percent solvent. The washed pectin is drained and pressed to remove up to 50 percent of the solvent and water present, and it is then dried on a heated drum to a moisture content of 6 to 10 percent. The dried pectin is ground to pass a 60 mesh screen, and packaged for marketing. Some citrus pectin is sold as a liquid product in solution with added citric acid. In an efficiently operated plant the apparent high cost of solvent is considerably reduced by employing solvent recovery throughout all stages of precipitation, washing and drying $[8,9,11,12]$. 


\section{Material and Methods}

The following material was used during laboratory work.

Knife, Crushers, Sieves, Digital pH meter, Beaker, Filter paper, Strainer with cheese cloth, Digital and non-digital driers or ovens, Water bath, Centrifuge and Refrigerator

\subsection{Raw Material Preparation}

Fresh orange waste consists of peel and pulp was collected from restaurant, hotels, pastries\& juice processing houses in local city. The fresh peel was then sorted, washed, dried, milled \& packed in air tight, moisture proof bag at room temperature until required for the extraction processes.

\subsection{Procedure of Experiment}

Orange peels were collected from juice house. It was collected in plastic bags and transported for analysis. The following methods were followed.

a. Sample collection was done

b. Oranges peel was prepared with 4 skin quarters

c. Quarters were cut into narrower strips and removed the white peel with a knife.

d. Then grated white peel was mixed with 2.5 and $1.75 \mathrm{ml}$ of HCL, and then 500 and $250 \mathrm{ml}$ of water was added respectively for each beaker.

e. Then it was cooked gently for 1-2hr. each pans.

f. After this the peels powder were removed from the extracts by using filter cheese cloth.

g. The extracted pectin was precipitated by adding equal ratio of ethanol to the extracted of pectin with thorough stirring, left for 30 minutes to allow the pectin float on the surface.

h. Gel pectin was separated from ethanol and water by centrifuge.

i. The extracted pectin was purified by washing in 200 $\mathrm{mL}$ acetone and then pressed on a nylon cloth to remove the residual $\mathrm{HCl}$ and universal salt.

j. The resulting pectin was weighed and shredded into small pieces and was air drier.

\subsection{Sample Preparation}

The sample acquired had to be prepared and conditioned for water bath heating, filtration, precipitation of pectin and centrifuge. Sample preparation process including manual size reduction (Knife cutting), drying, and milling and sieving after the samples were collected. Peels of orange 930gm are used for the sample preparation. They were cut by knife into pieces of about $3-5 \mathrm{~cm}$ length for drying and milling. The Sample was drying at temperature of $80^{\circ} \mathrm{C}$ for $(18-24) \mathrm{hr}$. After drying, each of the samples was milled separately [13].

\subsection{Pretreatment of Orange Peel Powder}

Pretreatment must meet the following requirements: improve the formation of sugar, avoid the degradation or loss of carbohydrate. The main purpose for pretreatment of orange peel is to destroy shell protecting cellulose, decrease crystallite of cellulose and must break this shell for enzyme to must break this shell for enzyme to access substrate (sugar) [13].

\subsection{Heating Pretreatment}

The powders of orange peel were treated inside water bath heating; every different sample was treated separately. Heating pretreatment used at $80^{\circ} \mathrm{C}$ temperatures and using the shaking water bath [11].

\subsection{Procedures in Heating Pretreatments}

The following procedures were used to conduct heating pretreatments [13].

a. $5 \mathrm{~g}$ of orange peel powder was added in to $1000 \mathrm{ml}$ conical flasks. Then $300 \mathrm{ml}$ of distilled water was added to beaker.

b. The conical flasks was capped with the help of rubber plugs, shaker substrate primarily after checking for $\mathrm{PH}$ using a digital $\mathrm{PH}$ meter. The $\mathrm{PH}$ then was adjusted to 1.96-2.1. Hydrochloric acid solution was added drop wise to the other flask with constant stirring until the $\mathrm{pH}$ reached to a range of 1.96-2.1.

c. Water bath was heated at a temperature of $80^{\circ} \mathrm{C}$ for (12) hours.

d. After finishing the given pretreatment time and temperature the sample in water was allowed to cool and separate soluble pectin from the insoluble by filtrate. The soluble pectin was precipitation of pectin in the next steps $\&$ the soluble solution in another $1000 \mathrm{ml}$ of beaker.

\subsection{Filtration}

The cake was removed from the extracts by using filter cheese cloth. After that the extracted pectin was precipitated by adding equal ratio of ethanol to the extracted of pectin with thorough stirring, left for 30 minutes to allow the pectin float on the surface [14].

\subsection{Centrifuge}

It was used to separate the gel pectin from ethanol and water. In addition to the extracted pectin was purified by washing in $200 \mathrm{~mL}$ acetone and then pressed on a nylon cloth to remove the residual $\mathrm{HCl}$ and universal salt [13].

\subsection{Dryer Pectin}

The washed pectin was drained and pressed to remove the solvent and water present, and it was then dried on a heated drum to a moisture content of 6 to 10 percent. The dried pectin was ground to pass a 60 mesh screen, and packaged for marketing. Some citrus pectin was sold as a liquid product in solution with added citric acid. In an efficiently operated plant the apparent high cost of solvent was considerably reduced by employing solvent recovery throughout all stages of precipitation, washing and drying [13]. 


\section{Result and Discussion}

\subsection{Characterization of Extracted Pectin}

The dried pectin obtained from the various peels of the oranges was subjected to the following characterization parameters.

a. Color: This was done by visual observation and the pectin is important as it affects the appearance of the gel produced. And it was observed to be white red.

b. Solubility of dry pectin in cold and hot water: The pectin samples were separately placed in a conical flask with $10 \mathrm{~mL}$ of $95 \%$ ethanol followed by $50 \mathrm{~mL}$ distilled water. The mixture was shaken vigorously to form a suspension which was then heated at $80^{\circ} \mathrm{C}$ for $15 \mathrm{~min}$. It has been observed to be soluble.

c. Sugar and organic acids: One gram of the pectin sample was placed separately in $500 \mathrm{~mL}$ flask each and moisture with $5 \mathrm{~mL}$ ethanol, $100 \mathrm{~mL}$ water poured rapidly, shaken and allowed to stand for 10 minutes. To this solution, $100 \mathrm{~mL}$ ethanol containing $0.3 \mathrm{~mL}$ hydrochloric acid was added, mixed and filtered rapidly, $2.5 \mathrm{~mL}$ of the filtrate was measured into a conical flask $(25 \mathrm{~mL})$, the liquid has evaporated on a water bath and the residue dried in an oven at $50^{\circ} \mathrm{C}$ for 2 hours. It has been checked as it is organic compound.

d. $\mathrm{PH}$ determination: The choice of the $\mathrm{pH}$ was made by preparing a buffer at $\mathrm{pH} 7.0$ and the temperature adjusted to $28^{\circ} \mathrm{C}$, the glass electrode standardized with standard buffer solution with the electrode rinsed with distilled water before inserting into the pectin solution and $\mathrm{pH}$ determined read off. It has been found that 5.0 of $\mathrm{pH}$ reading.

e. Equivalent weight determination: Pectin sample (5 g) was weighed into a $300 \mathrm{~mL}$ conical flask and moistened with $5 \mathrm{~mL}$ ethanol, was added to the mixture followed by $100 \mathrm{~mL}$ distilled water and few drops of phenol red indicator. Care was taken at this point to ensure that all the pectin had dissolved and that no clumping occurred at the sides of the flask before the solution was then slowly titrated (to avoid possible de-esterification).

The peel was pre-treated with absolute ethanol for 30 minutes. The reason is because ethanol peel pre-treatment improves purity by the removal of ethanol-soluble components, including sugars, and inactivates enzymes without leaching soluble pectin fractions. The peel was cut into the smallest size as the effect of size of the particles of the raw material. The pectin yield was reported to be significantly higher with the use of flour as raw material $(9.73 \%)$; when the extraction was made from the lemon the yield was $6.13 \%$. It proved that proto pectin is more available in small particles than in large one.

Fresh pressed peel was chosen for use in all extractions instead of dried peel. This decision was based on the loss of yield reported in use of dried peel in pectin extraction. A reduction in both pectin yield and pectin grade due to drying orange peel. Because the goal of this project was to optimize pectin yield, it was decided that fresh pressed peel would be used so that drying conditions would not cause a loss in pectin yield. A separate study could be conducted in the future to determine the optimal drying characteristics for orange peel [15].

Factorial experiments were not conducted for either extraction method due to the limited amount of peel available from a single orange source, the length of time necessary to conduct each individual trial, and the limited availability of experimental instrumentation and supplies that were necessary to conduct each trial. To conduct a factorial experiment with these limitations, fewer extraction parameters and levels within each parameter could be investigated. Instead, optimization was performed for each parameter individually and the best results were carried over into optimization of the next parameter [14].

\subsection{Acid Extraction Experiments}

Acid extractions were started utilizing a shaking water bath with magnetic spiritual Capability. All tests of extraction parameters and orange peel maturity and variety. The Precision shaking water bath with heating capability up to $80^{\circ} \mathrm{C}$ was desired for use due to its ability to hold extraction temperatures. Once the water bath was working, several of the optimization experiments were repeated utilizing the shaking water bath to determine if any variations would exist due to its use. The final comparison of acid extraction was conducted utilizing the water bath [13].

\section{Conclusions}

The extraction method used was efficient in estimating the yield of solutes for pectin production from the source. For all design points, the final product was estimated based on the total dissolved solutes concentration of the extract at equilibrium. The results of analysis of variance showed that the effects of the $\mathrm{pH}$, on the yield of the extracted solutes were found to be significant. The interaction of $\mathrm{pH}$ was also found to be significant, however to a lower extent. The response increased in the yield of pectin was observed when the variables $\mathrm{pH}$ and temperature were used at the $\mathrm{pH}=2.0$ and $80^{\circ} \mathrm{C}$. Moreover, it was also more efficient in approximating the appropriate volume of solvent used to extract a given mass of peel and ethanol efficiently. Based on the results of the experiment, the approximate volume of solvent used for the orange peel and ethanol. These results demonstrated the successful extraction of solutes for pectin production with hydrochloric acid, providing potential benefits to industrial extraction of pectin, from an economic as well as an environmental point of view.

\section{References}

[1] Van Buren, J. P. 2012. Function of pectin in plant tissue structure and firmness. In The Chemistry and technology of pectin. Academic Press. California. 
[2] Quebedeaux B, Bliss F. Horticulture and human health: contributions of oranges and vegetables. Proc. Symp. 1988. Hort. and Human Health Prentice.

[3] Tobias I. Ndubuisi Ezejiofor, N. V. Eke, R. I. Okechukwu, R. N. Nwoguikpe and C. M. Duru. (2017) Waste to wealth: Industrial raw materials potential of peels of Nigerian sweet orange (Citrus sinensis). African Journal of Biotechnology Vol. 10 (33).

[4] S. Wang, F. Chen, J. Wu, Z. Wang, X. Liao, X. Hu, Journal of Food Engineering, 2012, 78, 693-700.

[5] International Pectin Producers Association, 2016.

[6] Braddock, R. J. 2013. Handbook of citrus by-products and processing technology.

[7] May, C. 2014. Industrial pectin's: sources, production, and applications. Carbohydrate Polymers.

[8] Wargovich M. Anticancer properties of oranges, Hort Science. 2009. 35: 573-575.

[9] Kertesz, Z. I., Kim, H. and Fassihi, R., Application of a binary polymer system in drug release rate modulation, Journal of Pharmaceutical Sciences, 86, (1951) p. 316-322.
[10] Hercules, food gum product description: General description of pectin, 1999.

[11] kohn R, Binding toxic cations to pectin, its oligomer fragment and plant tissues, carbohydrate polym, 2002, 2, 273-275.

[12] Joye DD and Luzio GA, process for selective extraction of pectin's from plant material by differential $\mathrm{PH}$, carbohydrate polym, 2010, 43 (4), 337-342.

[13] Extraction of pectin by microwave heating pressure, United States patent.

[14] Crandall, P. G., R. J. Braddock, A. H. Rouse, 2015, Effect of drying on pectin made from Orange peel and lemon pomace, Journal of Food Science.

[15] Thermodynamic properties for characterizing ethanol and water.

[16] Coulson and Richardson's chemical engineering, volume 6. 